\title{
Optimal impulsive control with application to antiangiogenic tumor therapy
}

\author{
F. Cacace* ${ }^{*}$ V. Cusimano ${ }^{\dagger}$ P. Palumbo ${ }^{\dagger}$ \\ * f.cacace@unicampus.it, Università Campus Bio-Medico di Roma, Roma, Italy \\ $\dagger$ \{valerio.cusimano,pasquale.palumbo\}@iasi.cnr.it, IASI-CNR, Roma, Italy
}

\begin{abstract}
An optimal control algorithm is proposed for impulsive differential systems, i.e. systems evolving according to ordinary differential equations between any two control actions, occurring impulsively at discrete time instants. Measurements are as well acquired at discrete time instants. The model-based control law is conceived for medical and health-care frameworks and, indeed, is applied to synthesize a feedback antiangiogenic tumor therapy. To cope with unavailable or temporally sparse measurements, the control law benefits of a state observer properly designed for continuous-discrete systems by suitably exploiting recent results on observers for time-delay systems. The closed-loop algorithm is validated by building up an exhaustive simulation campaign on a population of virtual subjects, each sampled from a multivariate Gaussian distribution whose mean and covariance matrix are identified from experimental data taken from the literature. In silico results are encouraging and pave the way to further clinical verifications.
\end{abstract}

Index Terms-Optimal control, Impulsive control, Discretization of nonlinear systems, Tumor therapy.

\section{INTRODUCTION}

This paper aims at describing a framework for the closedloop control of medical treatments. It is well known that biomedical systems pose specific challenges to control techniques originally developed for traditional engineering areas, due to modeling uncertainties, parameter variability, frequency of measurements and constraints on the control. We investigate how new estimation and control approaches can be exploited to alleviate these problems and pave the way to the design of reliable and optimized feedback-based protocols in the medical practice. Specifically, we consider the problem of estimation and output feedback control of nonlinear continuous-time systems under the constraint that measurements are available at discrete time points, with possibly large intervals, and that controls have an impulsive nature. These assumptions are tailored to a potentially large set of situations in which both the monitoring actions and the drug administration follow a discrete-time pattern, with potentially large and distinct time intervals.

In the literature several results can be found about impulsive control [24], [37], [41], [44], [49], [51], [53], based on the theory of impulsive dynamical systems [50]. Impulsive differential systems have already been exploited in biomedical applications, for instance in applications to pulse vaccination strategies [2], [11], [48]. In order to deal with discrete-time measurements taken at sampling intervals different than those of the impulsive control we follow a different approach. The optimal output-based impulsive feedback control is obtained by using two new tools to bridge the gap between the continuous-time system and the discrete measurements and controls. From the one hand, discrete measurements are modeled as a continuous-time output affected by a time-varying delay, for which a suitable nonlinear state-observer is applied [9], [10], providing an exponential estimate of the whole state. On the other hand, the system in free evolution (i.e. among any two control impulses) is discretized by means of the Carleman linearization approach [29]. With respect to standard numerical integrations, the advantage is that the continuoustime nonlinear system is transformed into a discrete-time linear system of higher dimensions. Even if the resulting system is time-varying and its matrices depend on the state, it enjoys the property that its dynamics can be inverted in time, thus making easier to compute the optimal solution with respect to a quadratic index through a classical LQ approach. By using these two distinct tools it is relatively easy to extend the proposed methodology to other scenarios, like continuous-time measurements with impulsive control, or discrete-time measurements with continuous or piece-wise continuous infusion therapy. The use of a state observer allows to overcome the drawback of [7], where preliminary results on impulsive control where presented according to a complete knowledge of the state of the system.

We apply our approach to the optimal control problem of antiangiogenic tumor therapy. The scientific basis of antiangiogenic therapy of cancer is widely discussed in the medical literature, see for example the recent review in [38]. The original idea of antiangiogenic tumor therapy is due to Folkman [21] and is based on two general principles, namely that tumor growth is angiogenesis dependent and that antiangiogenic therapy targets genetically stable microvascular endothelial cells that are less prone to developing drug resistance. Actually, no resistance to antiangiogenic inhibitors has been observed in experimental cancer studies. On the other side, antiangiogenic therapy only reduces the tumor size, ideally to its maximum avascular size, and the cancer grows back when the treatment is halted. For this reason, antiangiogenic therapy is not sufficient as a stand-alone treatment, but can be used in combination with other traditional therapies like chemotherapy and radiotherapy. The interest in antiangiogenic therapy was vastly increased when the first broad-spectrum angiogenesis inhibitors angiostatin and endostatin were described in 1994 and 1997 by Folkman's group [4], [38], [42], [43]. However, clinic trials based on a soluble preparation of human recombinant endostatin didn't confirm the expectations, since 
tumor regression was not consistently observed in Phase I and Phase II clinical trials [19], [30]. Endostatin development was pursued in China and has been approved by the Chinese SFDA since 2005 for use in combination with chemotherapy [23], [38]. Arguably, while for cytotoxic agents the reduction of the tumor mass is a requirement for response, for antiangiogenic agents tumor arrest and growth inhibition may already be a valuable achievement [38]. In this sense, several further studies have investigated the "additive" effects of multiple antiangiogenic therapies [1], [25], [26], as well as novel antiangiogenic approaches [40] and the different effects of distinct drug administration protocols, i.e. bolus doses, lowdose metronomic regimen and continuous infusion therapy [5], [22], [26], [27].

Due to the complexity of the angiogenesis process, the long term perspective of effective implementation of antiangiogenic tumor therapies requires reliable quantitative modeling of the interplay between tumor growth and the development of its vascular network as well as of the action of angiogenic inhibitors [47]. Folkman and his coworkers developed a simple but largely influential mathematical model of this kind, a minimally parametrized and low-dimensional dynamical system that describes the vascular phase of tumor growth [25], usually referred to as the Hahnfeldt et al. model. The Hahnfeldt et al. model has been the subject of intense research in the bio-mathematical field during the last 15 years [13], [18], [28], [46], [47], [52]. In [12] some variants are discussed, as well as the mathematical characterization of treatments leading to tumor eradication. Antiangiogenic therapies has been studied from the point of view of optimal control both as standalone therapy [8], [17], [20], [34]-[36], [45] and as part of a combined therapy [16], [32], [33]. Specifically, [14], [15] derive interesting conclusions about the interplay between chemotherapy and anti-angiogenic therapy through an analysis based on variants of the Hahnfeldt et al. model. In the latter work stochasticity and irregularity of drug delivery is also faced. Overall, these works provide important contributions to clarify how an optimal dosing strategy can be designed, but they generally assume that continuous-time infusion is applicable and that the state of the system is completely known. Exceptions to the first assumptions include [12], where the first example of impulsive control theory for a model of vascularized solid tumor is provided, and the recent work [17], where it is supposed that therapy is administered in bolus doses with fixed rest periods. Also in these cases, the state of the system is assumed to be known. The Hahnfeldt et al. system includes two state variables, representing the tumor size and the vascular carrying capacity. Tumor size can be measured, even if in practice measurements cannot be taken continuously, but the carrying capacity is an abstraction that does not correspond to a measurable quantity. Even if [47] argues that for the Hahnfeldt et al. model the optimal control does not change much by varying the carrying capacity, it is of both theoretical and practical interest to develop an impulsive control strategy based on a convergent estimation of the true state from discrete measurements.

The proposed antiangiogenic tumor therapy is validated by means of a simulation campaign carried out on a population of virtual subjects, each identified by a set of the Hahnfeldt et al. model parameters, properly sampled from a multivariate Gaussian distribution whose average value and covariance matrix are estimated from experimental data achieved from the literature. Because of the usually large inter- and intra-subjects variability affecting biological and physiological systems, we choose to design the control parameters on the average subject and keep them fixed when applied to the virtual subjects of a rather heterogenous population. Numerical simulations highlight (i) the efficacy of the designed closed-loop control law (in terms of a consistent reduction of the tumor size at the end of the period of drug administration) and (ii) the reliability of the therapy (validated by the boundedness of the maximum daily drug administration), paving the way to further clinical verifications.

The paper is organized as follows. Section II provides a description of the proposed methodology in a general framework, including the problem setting and the development of the optimal impulsive control algorithm. Section III is concerned with the application of the optimal impulsive control to the antiangiogenic tumor therapy, including a brief description of the tumor growth model under consideration. Performance analysis is reported in Section IV. Conclusions follow.

\section{MethodologicAl FRAMEWORK}

\section{A. Notation}

The symbol $\otimes$ denotes the Kronecker product. $M^{[i]}$ is the $i$-th Kronecker power of the matrix $M$, recursively defined by $M^{[i]}=M \otimes M^{[i-1]}$ and $M^{[0]}=1$. Given $f \in C^{\infty}\left(\mathbb{R}^{a} ; \mathbb{R}^{b}\right)$, and $x=\left[x_{1}, \ldots, x_{a}\right]^{T} \in \mathbb{R}^{a}, \nabla_{x} \otimes f$ is the function $\mathbb{R}^{a} \rightarrow$ $\mathbb{R}^{b \times a}$ defined by $\left[\partial f / \partial x_{1} \ldots \partial f / \partial x_{a}\right] . \nabla_{x}^{[i]} \otimes f$ denotes the same operation repeated $i$ times and it is a function $\mathbb{R}^{a} \rightarrow$ $\mathbb{R}^{b \times a^{i}}$ with $\nabla_{x}^{[0]} \otimes f=f$. Given the vector field $f$ and a scalar function $h, L_{f} h$ denotes the Lie derivative of $h$ with respect to the vector field $f$, defined as $L_{f} h(x)=\left(\nabla_{x} \otimes h\right)(x) \cdot f(x)$. $L_{f}^{i} h(x)$ denotes the $i$-th Lie derivative of $h$ with respect to the vector field $f$, with $L_{f}^{0} h(x)=h(x)$.

\section{B. Problem setting and state of the art}

Consider a strictly increasing and positive sequence of time instants $\mathcal{T}=\left\{\tau_{k}, k \in \mathcal{I}, \tau_{k+1}>\tau_{k} \geq 0\right\}$, with $\mathcal{I}=$ $\{0,1, \ldots, N\}$, and an impulsive differential system [31] with impulses occurring at times $\tau_{k} \in \mathcal{T}, k \in \mathcal{I}_{0}=\{1, \ldots, N\}$,

$$
\begin{aligned}
\dot{z}(t) & =f(z(t)), \quad t \in\left[\tau_{k}, \tau_{k+1}\right), \quad k \in \mathcal{I} \backslash\{N\} \\
z\left(\tau_{k}^{+}\right) & =z\left(\tau_{k}^{-}\right)+B v_{k}, \quad k \in \mathcal{I}_{0}
\end{aligned}
$$

where $\tau_{0}$, is the initial time instant, $z(t) \in \mathbb{R}^{n}, v_{k} \in \mathbb{R}^{p}$ and $f: \mathbb{R}^{n} \rightarrow \mathbb{R}^{n}$ is an analytic map. The initial condition is $z\left(\tau_{0}\right)=z_{0}$. In the sequel we will denote with $\sigma_{k}=\tau_{k+1}-$ $\tau_{k}>0$ the intervals between two consecutive impulses, and with $x_{k}=z\left(\tau_{k}^{+}\right)$the state of system at the discrete time when the impulsive input is delivered.

Measurements are acquired at fixed time instants in $\mathcal{S}=$ $\left\{s_{m}, m \in \mathcal{I}_{0}^{\prime}, s_{m+1}>s_{m} \geq 0\right\}$, with $\mathcal{I}_{0}^{\prime}=\left\{1, \ldots, N^{\prime}\right\}$ and

$$
y_{m}=h\left(z\left(s_{m}\right)\right)
$$


where $h: \mathbb{R}^{n} \rightarrow \mathbb{R}$ is an analytic map. In case $s_{m} \in \mathcal{T}$, then measurements are supposed to exclude the impulsive input, that is:

$$
y_{m}=h\left(z\left(s_{m}^{-}\right)\right) .
$$

For simplicity, we refer to the case of a scalar output, but the theory can be straightforwardly extended to deal with a vector output [10].

The following two working hypotheses are here investigated from a theoretical viewpoint. One case refers to input impulses administered more frequently than sampled measurements, that is, $\mathcal{S} \subseteq \mathcal{T}$. The other case refers to the opposite framework, with $\mathcal{T} \subseteq \mathcal{S}$. The former is the more common case, since often measurements are more difficult, time-consuming and expensive to obtain than control actuation.

A key point in the applications of optimal control to biomedicine is to provide a biologically meaningful control objective. Of course, the definition of this objective may depend on the specific problem or therapy and it will result in a cost function to be minimized that can assume different forms. The basic assumptions that we have followed in this work are partly tailored to the problem of antiangiogenic tumor therapy but in our view they can be shared by several similar applications:

- The objective of the control law is formulated on the discrete-time scale provided by the impulsive inputs and the measurements. The time intervals between these events usually depend on clinical practices that are not part of the control strategy itself, thus they are assumed as given but known. The time-scale is therefore a given and known parameter of the problem that cannot be changed.

- The therapy has a known time horizon. The objective of the control is to drive the state of the system to a desired value at the end of the therapy and, at the same time, to minimize the amount of the control input. This is a fairly common setting for a therapy, since the control input is usually a drug and its dose cannot assume arbitrarily large values. This assumption results in a cost function having two terms, the first one related to the distance from the desired final value and the other one related to the amount of the administered drug.

- Since our approach aims at transforming the original nonlinear continuous-time systems into a linear discretetime systems with impulsive controls, we formulate the cost terms as a quadratic function of the control pulses. In this way we can build upon known methods for the LQ (Linear Quadratic) optimal control framework.

Based on the above assumptions the aim of the proposed control action is to properly dose the impulsive inputs in order to minimize the following quadratic index:

$$
J\left(x_{N}, \mathbf{v}\right)=\left(x_{N}-\bar{x}\right)^{T} Q_{N}\left(x_{N}-\bar{x}\right)+\sum_{k=1}^{N} v_{k}^{T} R_{k} v_{k}
$$

where $Q_{N} \geq 0, R_{k}>0$, are weight matrices, $\mathbf{v}=$ $\left[v_{1}, \ldots, v_{N}\right]^{T}$ is the sequence of control impulses and $\bar{x}$ is the chosen reference level for the state of the system at the end of the control application (e.g., at the end of the period of drug administration therapy in case of medical/healthcare frameworks). Such a problem has been addressed in [7], by means of the Carleman-based discretization of the original continuous-time system at the time instants $\tau_{k} \in \mathcal{T}$ related to the impulsive control. According to [7], the discretized version of (1) is:

$$
x_{k+1}=\Psi_{k}\left(x_{k}, v_{k+1}\right), \quad x_{0}=z_{0}, \quad k \in \mathcal{I} \backslash\{N\}
$$

where

$$
\Psi_{k}\left(x_{k}, v_{k+1}\right)=x_{k}+F_{k}\left(x_{k}\right)+B v_{k+1}
$$

with

$$
F_{k}\left(x_{k}\right)=\sum_{j=0}^{\infty} P_{j}^{1}\left(x_{k}\right) \frac{\sigma_{k}^{j+1}}{(j+1) !} .
$$

$P_{j}^{1}$ belongs to the family of vectors $\left\{P_{j}^{i} \in \mathbb{R}^{n^{i}},(i, j) \in \mathbb{N}_{0} \times\right.$ $\mathbb{N}\}$, recursively defined by

$$
P_{j}^{1}=\sum_{l=1}^{j} A_{l}^{1} P_{j-1}^{l}, \quad P_{j}^{i}=\sum_{l=\max \{1, i-1\}}^{j} A_{l-i+1}^{i} P_{j-1}^{l}
$$

with $P_{0}^{1}=f, P_{\rho}^{i}=0$ for $i \geq 2$ and the matrix coefficients $A_{j}^{i} \in \mathbb{R}^{n^{i} \times n^{j+1-1}},(i, j) \in \mathbb{N}_{0} \times \mathbb{N}$, provided by

$$
\begin{aligned}
A_{j}^{1}(x) & =\frac{\left(\nabla_{z}^{[j]} \otimes f\right)(x)}{j !}, \\
A_{j}^{i} & =A_{j}^{1} \otimes I_{n}^{[i-1]}+I_{n} \otimes A_{j}^{i-1} .
\end{aligned}
$$

The Carleman embedding provides the exact solution to the discretization problem, whose finite-dimensional implementation is based on a suitable truncation of the involved series expansion (7).

Once the control optimization problem is set on a pure discrete-time framework, the optimal impulsive control problem is dealt with by solving the following constraints:

$$
\begin{array}{lll}
k \in \mathcal{I}_{0}: & \nabla_{v_{k}} \otimes \mathcal{L}=2 v_{k}^{T} R_{k}+\lambda_{k}^{T}\left(\nabla_{v_{k}} \otimes \Psi_{k-1}\right)=0, \\
k \in \mathcal{I}_{0}: & \nabla_{\lambda_{k}} \otimes \mathcal{L}=\Psi_{k-1}-x_{k}=0, \\
k \in \mathcal{I}_{0} \backslash\{N\}: & \nabla_{x_{k}} \otimes \mathcal{L}=\lambda_{k+1}^{T}\left(\nabla_{x_{k}} \otimes \Psi_{k}\right)-\lambda_{k}^{T}=0, \\
k=N: & \nabla_{x_{k}} \otimes \mathcal{L}=2\left(x_{k}-\bar{x}\right)^{T} Q_{N}-\lambda_{k}^{T}=0 .
\end{array}
$$

where $\Psi_{k}$ denotes $\Psi_{k}\left(x_{k}, v_{k+1}\right)$ for short, and $\mathcal{L}$ is the Lagrangian

$$
\mathcal{L}(\mathbf{x}, \Lambda, \mathbf{v})=J\left(x_{N}, \mathbf{v}\right)+\sum_{k=0}^{N-1} \lambda_{k+1}^{T}\left(\Psi_{k}-x_{k+1}\right),
$$

with $\mathbf{x}=\left[x_{1}^{T}, \cdots, x_{N}^{T}\right]^{T}$ and $\Lambda=\left[\lambda_{1}, \ldots, \lambda_{N}\right]^{T}, \lambda_{i} \in \mathbb{R}^{n}$ is the vector of Lagrangian multipliers.

The solution is given by

$$
v_{k}=\frac{1}{2} R_{k}^{-1} B^{T} \lambda_{k}
$$

with $x_{1}, \ldots, x_{N}, \lambda_{1}, \ldots, \lambda_{N} \in \mathbb{R}^{n}$ available by numerically solving the usual backward/forward equations of the constraints.

From a mathematical viewpoint this formulation yields a system of $2 n N$ nonlinear equations with respect to the unknowns $\mathbf{x}, \Lambda$. Since the numerical computation of the solutions is computationally challenging, two possible methods can be 
pursued. The first one is to resort to receding horizon algorithms that allow to decompose the original numerical problem on a set of reduced order similar tasks. The second method is to exploit the structure of the Carleman discretization that allows to write the system equations backward in time. Indeed, the Carleman backward discretization starts with the "initial point" $x_{k+1}-B v_{k+1}$ and backwardly provides the "final point" $x_{k}$ as

$$
x_{k}=x_{k+1}-B v_{k+1}+\sum_{j=0}^{\infty} P_{j}^{1}\left(x_{k+1}-B v_{k+1}\right) \frac{\left(-\sigma_{k}\right)^{j+1}}{(j+1) !} .
$$

Therefore, both $\Lambda$ and $\mathrm{x}$ can be written backward as functions of the only unknown $x_{N}$, thus reducing the optimization task to the search for $x_{N}$ only (passing from $2 n N$ to $n$ unknowns).

Indeed, both these approaches have been followed in [7], under the assumption of complete knowledge of the initial state of the system. Unfortunately, such assumption is not realistic, especially in biomedical applications where only few compartments are accessible for measurements. We overcome this drawback by means of a state observer that allows to estimate the state of the system by properly exploiting the available sampled measurements.

\section{Optimal impulsive control algorithm}

As previously stated, the optimal impulsive control algorithm developed in [7] (and briefly recap in the previous Subsection) requires the knowledge of the initial state $x_{0}$. The proposed control algorithm overcomes such requirement of complete knowledge of the state by means of a state-observer whose equations are updated by a discrete stream of sampled measurements (see next Subsections for the observer details). In the following the observer-based state estimate computed at time $\tau_{k}$ will be denoted by $\hat{x}_{k}$.

Case $\mathcal{S} \subseteq \mathcal{T}$. In case the control impulses are more frequent than the measurements, there exists an injection $k: \mathcal{I}_{0}^{\prime} \mapsto \mathcal{I}_{0}$, $k=\nu(m)$ that allows to write $s_{m}=\tau_{\nu(m)} \in \mathcal{T}$, for any $m \in \mathcal{I}_{0}^{\prime}$.

The initialization of the algorithm substantially requires the computation of the solution of the optimal control problem by substituting the initial estimate $\hat{x}_{0}$ in (4)-(13) in place of the real state $x_{0}$. This solution $\mathbf{v}$ does not consider any subsequent measurement, and we apply it only regards to the the control impulses $v_{1}, \ldots, v_{\nu(1)}$ (i.e the first $\nu(1)$ entries of $\mathbf{v}$ ). Meanwhile, the observer evolves time-continuously in $\left[\tau_{0}, s_{1}\right)$, providing at $t=s_{1}$ the update of the state estimate $\hat{x}_{\nu(1)}$. Notice that this initial run of the observer trivially predicts the state evolution without the correction term since no measurements are available in $\left[\tau_{0}, s_{1}\right)$ yet. Then, at $t=s_{1}$ the optimization control problem (4)-(13) is reformulated in $\left[\tau_{\nu(1)}, \tau_{N}\right]$ with respect to the new initial state $\hat{x}_{\nu(1)}$. This solution provides the control impulses $\left\{v_{\nu(1)+1}, \ldots, v_{N}\right\}$, but we keep (and apply) the impulses on the time instants $\left\{\tau_{\nu(1)+1}, \ldots, \tau_{\nu(2)}\right\}$. Meanwhile the observer evolves time-continuously in $\left[s_{1}, s_{2}\right)$, with the benefit of the new measurement acquired at time $t=s_{1}$, providing at $t=s_{2}$ the update of the state estimate $\hat{x}_{\nu(2)}$. And so on.
The optimization procedure is below formalized.

\section{Control Algorithm A1.}

First interval $\left[\tau_{0}, s_{1}\right)$ :

0) the initial estimate $\hat{x}_{0}$ is set;

1) the optimization problem (4)-(13) is solved for the time interval $\left[\tau_{0}, \tau_{N}\right]$, with respect to the initial state $\hat{x}_{0}$, providing the control inputs $\left\{v_{1}, \ldots, v_{N}\right\}$;

2) we apply only the input impulses $\left\{v_{1}, \ldots, v_{\nu(1)}\right\}$;

3) the observer evolves in free evolution in $\left[\tau_{0}, s_{1}\right)$. At $t=$ $s_{1}$ it provides the estimate $\hat{x}_{\nu(1)}$.

Intervals $\left[s_{m}, s_{m+1}\right), m=1, \ldots, N^{\prime}-1$ :

i) the estimate $\hat{x}_{\nu(m)}$ is available from the observer; a new measurement $y_{m}$ at time $s_{m}$ is acquired;

ii) the optimization problem (4)-(13) is solved for the time interval $\left[\tau_{\nu(m)}, \tau_{N}\right]$, with respect to the initial state $\hat{x}_{\nu(m)}$, providing the control inputs $\left\{v_{\nu(m)+1}, \ldots, v_{N}\right\}$;

iii) we apply only the input impulses $\left\{v_{\nu(m)+1}, \ldots, v_{\nu(m+1)}\right\}$;

iv) the observer evolves in $\left[s_{m}, s_{m+1}\right)$, with the benefit of the last measurement $y_{m}$. At $t=s_{m+1}$ it provides the estimate $\hat{x}_{\nu(m+1)}$.

v) if $m<N^{\prime}-1$, update $m \mapsto m+1$ and go to item i)

Last interval $\left[s_{N^{\prime}}, \tau_{N}\right)$ (in case $\tau_{\nu\left(N^{\prime}\right)}<\tau_{N}$ ):

- the estimate $\hat{x}_{\nu\left(N^{\prime}\right)}$ is available from the observer;

- the optimization problem (4)-(13) is solved for the time interval $\left[\tau_{\nu\left(N^{\prime}\right)}, \tau_{N}\right]$, with respect to the initial state $\hat{x}_{\nu\left(N^{\prime}\right)}$, providing the control inputs $\left\{v_{\nu\left(N^{\prime}\right)}, \ldots, v_{N}\right\}$, and we apply all the input impulses.

The optimization time intervals reduce their length at each new step (from $\left[\tau_{0}, \tau_{N}\right]$, to $\left[\tau_{\nu(1)}, \tau_{N}\right]$, till $\left[\tau_{\nu\left(N^{\prime}\right)}, \tau_{N}\right]$ ), that means an algebraic system of $2 n(N-\nu(m))$ nonlinear equations is required to be solved at each step related to the generic interval $\left[\tau_{\nu(m)}, \tau_{N}\right]$.

Unfortunately, the computational task is still cumbersome and motivates the search for receding horizon procedures as the one proposed below.

Define $r_{k}=\varphi\left(\tau_{k}\right) \in \mathbb{R}^{n}, \tau_{k} \in \mathcal{T}$, a discrete monotonic trajectory, definitely approaching the desired final value $\bar{x}=$ $r_{N}$. These points can be thought of as a sequence of desired intermediate values for the state. Let $M<N$ be the number of reduced steps related to the chosen receding horizon approach. Then, Control Algorithm A1 is modified as follows, with the algorithm updating in $\left[\tau_{k}, \tau_{k+1}\right)$ (instead of in $\left[s_{m}, s_{m+1}\right)$ ).

\section{Control Algorithm A2:}

0) the estimate $\hat{x}_{k}$ is available from the observer; if there exists an $m \in \mathcal{I}_{0}^{\prime}$ such that $k=\nu(m)$, then a new measurement $y_{m}$ is acquired;

1) the optimization problem (4)-(13) is solved for the time interval $\left[\tau_{k}, \tau_{k+M}\right]$, with respect to the initial state $\hat{x}_{k}$, according to the desired final value $\bar{x}$ replaced by $r_{k+M}$. Such solution provides the control inputs $\left\{v_{k+1}, \ldots, v_{k+1+M}\right\}$;

2) we apply only the first input impulse $v_{k+1}$;

3) the observer evolves in $\left[\tau_{k}, \tau_{k+1}\right)$. At $t=\tau_{k+1}$ it provides the estimate $\hat{x}_{k+1}$. 
Clearly, the backward Carleman discretization can be applied on top of the receding horizon approach, thus further lightening the computational effort.

Case $\mathcal{T} \subseteq \mathcal{S}$. In case the control inputs are less frequent than the measurements, there exists an injection $m: \mathcal{I}_{0} \mapsto \mathcal{I}_{0}^{\prime}$, $m=\mu(k)$, that allows to write $\tau_{k}=s_{\mu(k)} \in \mathcal{S}$, for any $k \in \mathcal{I}_{0}$. The following algorithm benefits of the state estimates in time instants $t$ different than of the control input instants (i.e. $t$ not in $\mathcal{T}$ ): these estimates will be denoted by $\hat{z}(t)$. The initial estimate $\hat{x}_{0}$ is exploited to preliminary run the observer in $\left[\tau_{0}, s_{\mu(1)-1}\right)$ in order to achieve the state estimate at $t=s_{\mu(1)-1}$, right before the application of the first control impulse. Dealing with an asymptotic observer such estimate is supposed to be closer to the real state with respect to an a priori initialization at $t=\tau_{0}$, since we exploit the measurements at $t \in\left\{s_{1}, \ldots, s_{\mu(1)-2}\right\}$. Then, the optimization algorithm (4)-(13) is applied in $\left[s_{\mu(1)-1}, \tau_{N}\right]$, providing the control sequence $\mathbf{v}$, according to which we keep (and apply) only the first $v_{1}$ in $\tau_{1}$. Then we run the observer in $\left[s_{\mu(1)-1}, s_{\mu(2)-1}\right)$, in order to update the new initial estimate in $t=s_{\mu(2)-1}$ right before the application of the second control impulse. Then, we run the optimization algorithm in $\left[s_{\mu(2)-1}, \tau_{N}\right]$. And so on. The optimization procedure is below resumed.

\section{Control Algorithm A3:}

First interval $\left[\tau_{0}, s_{\mu(1)-1}\right)$ :

$0)$ The observer evolves in $\left[\tau_{0}, s_{\mu(1)-1}\right)$ (with the initial estimate $\hat{x}_{0}$ ) providing at time $s_{\mu(1)-1}$ the estimate $\hat{z}\left(s_{\mu(1)-1}\right)$ that benefits of the measurements at times $t \in\left\{s_{1}, \ldots, s_{\mu(1)-2}\right\}$;

Intervals $\left[s_{\mu(k)-1}, s_{\mu(k+1)-1}\right), k=1, \ldots, N-1$ :

i) the estimate $\hat{z}\left(s_{\mu(k)-1}\right)$ is available from the observer;

ii) the optimization problem (4)-(13) is solved for the time interval $\left[s_{\mu(k)-1}, \tau_{N}\right]$, with respect to the initial state $\hat{z}\left(s_{\mu(k)-1}\right)$, providing the control inputs $\left\{v_{k}, \ldots, v_{N}\right\}$;

iii) we apply only the first input impulse $v_{k}$;

iv) the observer evolves in $\left[s_{\mu(k)-1}, s_{\mu(k+1)-1}\right)$, with the benefits of the measurements acquired in $\left[s_{\mu(k)}, s_{\mu(k+1)-2}\right]$. At $t=s_{\mu(k+1)-1}$ it provides the estimate $\hat{z}\left(s_{\mu(k+1)-1}\right)$.

v) if $k<N-1$, update $k \mapsto k+1$ and go to item $i$ ).

Similarly to the case of $\mathcal{S} \subseteq \mathcal{T}$ such an optimization procedure is cumbersome and receding horizon procedures are suggested. To this end, recalling the role of $r_{k}=\varphi\left(\tau_{k}\right)$, let $M<N$ be the number of reduced steps related to the chosen receding horizon approach. Then, the Control Algorithm A3 is modified as follows, with the algorithm still updating in $\left[s_{\mu(k)-1}, s_{\mu(k+1)-1}\right)$.

\section{Control Algorithm A4:}

i) the estimate $\hat{z}\left(s_{\mu(k)-1}\right)$ is available from the observer;

ii) the optimization problem (4)-(13) is solved for the time interval $\left[s_{\mu(k)-1}, \tau_{k+M-1}\right]$, with respect to the initial state $\hat{z}\left(s_{\mu(k)-1}\right)$, according to the desired final value $\bar{x}$ replaced by $r_{k+M-1}$. Such solution provides the control inputs $\left\{v_{k}, \ldots, v_{k+M}\right\}$;

iii) we apply only the first input impulse $v_{k}$; iv) the observer evolves in $\left[s_{\mu(k)-1}, s_{\mu(k+1)-1}\right)$, with the benefits of the measurements acquired in $\left[s_{\mu(k)-1}, s_{\mu(k+1)-2}\right]$. At $t=s_{\mu(k+1)-1}$ it provides the estimate $\hat{z}\left(s_{\mu(k+1)-1}\right)$.

Also in this case the backward Carleman discretization can be applied to the receding horizon approach, to unburden the computational effort.

\section{State observer for continuous-discrete systems}

The observer exploited in the control algorithm is the one developed in [9], [10] and is based on the idea to model the discrete-time output as a continuous-time stream of data with a time-varying delay:

$$
Y_{m}(t)=h\left(z\left(t-\theta_{m}(t)\right)\right), \quad t \in\left[s_{m}, s_{m+1}\right),
$$

with $\theta_{m}(t)=t-s_{m} \in\left[0, s_{m+1}-s_{m}\right)$ a time-varying delay bounded by $s_{m+1}-s_{m}$. Clearly, by definition, it is:

$$
Y_{m}(t) \equiv y_{m}=h\left(z\left(s_{m}\right)\right), \quad t \in\left[s_{m}, s_{m+1}\right) .
$$

In this way, an observer for nonlinear systems endowed with continuous-time output measurements affected by timevarying delays can be applied to obtain a continuous-time estimation from discrete measurements. The observer in [9], [10] requires a drift-observability condition, that means the drift-observability map $\Theta(z)$ defined by

$$
\xi=\Theta(z)=\left[\begin{array}{c}
h(z) \\
L_{f} h(z) \\
\vdots \\
L_{f}^{n-1} h(z)
\end{array}\right]
$$

is a diffeomorphism in the domain of interest $D$ and $\Theta, \Theta^{-1}$ are uniformly Lipschitz in $D$ (if $D \equiv \mathbb{R}^{n}$ the system is said GULDO, i.e. Globally Uniformly Lipschtiz Drift-Observable). If that is the case, denoted by $\hat{z}(t)$ the state estimate provided by the observer, then for any $\alpha>0$ there exists an observer gain matrix $K$ and a delay bound $\bar{\theta}$ such that the observer equations defined on $t \in\left[s_{m}, s_{m+1}\right), m \in \mathcal{I}_{0}^{\prime}$

$$
\begin{aligned}
\dot{\hat{z}}(t) & =f(\hat{z}(t)) \\
& +J_{\Theta}(\hat{z}(t))^{-1} e^{-\alpha \theta_{m}(t)} K\left(Y_{m}(t)-h\left(\hat{z}\left(t-\theta_{m}(t)\right)\right)\right),
\end{aligned}
$$

with $\hat{z}\left(s_{m}\right)$ as initial condition ensures an exponential error decay rate $\alpha$ if $t-s_{m} \leq \bar{\theta}$. Notice that the latter condition is equivalent to having $s_{m+1}-s_{m} \leq \bar{\theta}$, that is, the measurement intervals are uniformly bounded by $\bar{\theta} . J_{\Theta}(\hat{z})$ is the Jacobian matrix of the drift-observability map $\Theta(z)$.

According to the impulsive fashion of the control input, whenever it is applied in $t=\tau_{k}, k \in \mathcal{I}_{0}$, the state estimate is updated as follows

$$
\begin{aligned}
\hat{x}_{k} & =\hat{z}\left(\tau_{k}^{+}\right) \\
\hat{z}\left(\tau_{k}^{+}\right) & =\hat{z}\left(\tau_{k}^{-}\right)+B v_{k}
\end{aligned}
$$




\section{ANTIANGIOGENIC TUMOR THERAPY}

\section{A. Model of the antiangiogenic therapy}

The Hahnfeldt et al. model here adopted belongs to the family of models introduced in [25], and is based on ordinary differential equations accounting for angiogenic stimulation and inhibition. Under the hypothesis that tumor growth is strictly dependent on the development of its vasculature, the dynamics of the tumor size is modeled as a Gompertz growth law with a dynamically varying term that models the development of the vasculature, the vasculature carrying capacity. On the other hand, the growth rate of the vasculature will be antagonized by endogenous factors and by the presence of anti-angiogenic drug. A classification of tumor growth models can be found in [3].

The Hahnfeldt et al. model and its variants are very popular and have been thoroughly discussed in many publications, see for example [12], [13], [47]. Controllability issues for this class of models have been studied in [28], [52].

In this paper we refer to the following version of the Hahnfeldt et al. model

$$
\begin{aligned}
& \dot{z}_{1}=-\lambda z_{1} \ln \left(\frac{z_{1}}{z_{2}}\right), \\
& \dot{z}_{2}=b z_{1}-\left(\mu+d z_{1}^{2 / 3}\right) z_{2}-c z_{2} z_{3},
\end{aligned}
$$

where $z_{1},\left[\mathrm{~mm}^{3}\right]$, denotes the tumor volume and $z_{2},\left[\mathrm{~mm}^{3}\right]$ is the vasculature carrying capacity. In particular, the first equation describes the evolution of the tumor size, that depends on the tumor growth rate $\lambda,\left[\mathrm{day}^{-1}\right]$. The second equation refers to the carrying capacity dynamics, with a production rate provided by the stimulatory capacity of the tumor upon the inducible vasculature $\left(b z_{1}\right.$ with $b$, [day $\left.{ }^{-1}\right]$, the vascular birth rate), and a clearance rate split in three terms: a spontaneous loss $\left(\mu z_{2}\right.$ with $\mu$, $\left[\right.$ day $^{-1}$ ], the rate of the spontaneous vascular inactivation), an endogenous inhibition which depends on the tumor volume $\left(d z_{1}^{2 / 3} z_{2}\right.$ with $d$, [day ${ }^{-1} \mathrm{~mm}^{-2}$ ], the endothelial cell death) and a third contribute depending on the exogenous drug administration and providing a vasculature inhibitory action $\left(c z_{2} z_{3}\right.$ with $c$, $\left[\right.$ day $\left.^{-1}(\mathrm{mg} / \mathrm{kg})^{-1}\right]$, the sensitivity to the drug). $z_{3},[\mathrm{mg} / \mathrm{kg}]$, denotes the serum level of the administered angiogenic inhibitor.

Following the literature [25], the value of parameter $\mu$ is assumed to be zero in the following, because it was found to be negligible for this system (i.e., constitutive endothelial cell loss does not play a major role).

Since the antiangiogenic drug is not directly administered in vein a further compartment is considered to account for drug diffusion,

$$
z_{3}(t)=\int_{0}^{t} e^{-\eta\left(t-t^{\prime}\right)} u\left(t^{\prime}\right) d t^{\prime},
$$

where $u,\left[\mathrm{day}^{-1}(\mathrm{mg} / \mathrm{kg})\right]$ is the actual drug infusion rate, i.e. the control law, and $\eta,\left[\mathrm{day}^{-1}\right]$, is the degradation rate constant. Eq.(21) can be written in its differential form as

$$
\dot{z}_{3}=-\eta z_{3}+u \text {. }
$$

A thorough mathematical analysis concerning the qualitative behavior of the present model (and of the more general family of models describing the mutual interaction between tumour growth and vasculature) can be found in [13], where conditions are given according to which tumor eradication is achieved for specific "open loop" control strategies, such as constant or periodic infusion therapies.

If we assume that (i) the input is administered in pulses, that (ii) the only available measurement, exploited to design the control law, is the size of the tumor, that is, the first component of the state vector $z$, and that (iii) these measurements are acquired at discrete sampling times, then system (20)-(22) can be represented according to the formalism of (1) (and will be referred to in the sequel as the impulsive Hahnfeldt et al. model') where

$$
\begin{aligned}
& f(z)=\left[\begin{array}{c}
-\lambda z_{1} \ln \left(\frac{z_{1}}{z_{2}}\right) \\
b z_{1}-d z_{1}^{2 / 3} z_{2}-c z_{2} z_{3} \\
-\eta z_{3}
\end{array}\right] \quad B=\left[\begin{array}{l}
0 \\
0 \\
1
\end{array}\right], \\
& h(z)=C z=z_{1} \quad \text { with } \quad C=\left[\begin{array}{lll}
1 & 0 & 0
\end{array}\right] .
\end{aligned}
$$

Differently from the continuous-time input $u(t)$ in (22), $v_{k}$ is expressed in $[\mathrm{mg} / \mathrm{kg}$ ] (it is an administered 'mass' instead of a 'rate' of administration, [( $\mathrm{mg} / \mathrm{kg}) /$ day], as in (22)).

The Hahnfeldt et al. model is conceived to provide, in absence of a therapy and assuming the initial carrying capacity is larger than the tumor size, a monotonic increase of tumor volume and carrying capacity to a unique equilibrium point $z_{1}=z_{2}=X_{\max }=(b / d)^{3 / 2}, z_{3}=0$ which is globally asymptotically stable [12], [25]. $X_{\max }$ may be thought of as an upper bound for both tumor and vasculature. A lower bound is naturally given by $\epsilon_{z}$, the size at which the tumor does not need any vasculature. As a matter of fact, a meaningful initialization of the type $z_{2}(0)>z_{1}(0)>\epsilon_{z}, z_{3}(0)=0$ and a proper choice of the final tumor target $\bar{x}$ such that $\epsilon_{z}<\bar{x}<X_{\max }$ allow to set $D=\left[\epsilon_{z}, X_{\max }\right] \times\left[\epsilon_{z}, X_{\max }\right] \times\left[0, \bar{z}_{3}\right]$ as the natural domain of interest for the Hahnfeldt et al. model, with $\bar{z}_{3}$ depending on the upper bound of the input $v$. In [8] it has been shown that, according to a meaningful choice of the model parameters and to the aforementioned setting of the initial conditions, the domain $D$ is an invariant set for the Hahnfeldt et al. model.

\section{B. Observer synthesis}

In order to design the continuous-time observer from discrete measurements for the impulsive Hahnfeldt et al. model (1) we need the following steps: (i) verify the Global Uniformly Lipschitz Drift-Observability hypothesis for (1) in the domain of interest $D$; (ii) design the observer gain $K$ in order to obtain a suitable convergence rate $\alpha$ for the control application, providing a delay bound $\bar{\theta}$ compatible with the maximum inter-sampling measurement interval, that means:

$$
\max _{m=1, \ldots N^{\prime}-1}\left\{s_{m+1}-s_{m}\right\} \leq \bar{\theta} .
$$

Regards to the first point, the drift-observability map (17) for $f$ and $h$ defined in (23)-(24) is

$$
\xi=\Theta(z)=\left[\begin{array}{c}
z_{1} \\
-\lambda(1+\log \tilde{z}) \\
\lambda z_{1}\left(\lambda(1+\log \tilde{z}) \log \tilde{z}+b \tilde{z}-d z_{1}^{2 / 3}-c z_{3}\right)
\end{array}\right]
$$


where $\tilde{z}=\frac{z_{1}}{z_{2}}$. The GULDO hypothesis requires the Jacobian matrix $J_{\Theta}(z)=d \Theta / d z$ to be non-singular in $D$, which is true because $\operatorname{det}\left(J_{\Theta}\right)=-c \lambda^{2} z_{1} \tilde{z}$ is not zero in $D$. The inverse $\Theta^{-1}$ of $\Theta$ is

$$
\begin{aligned}
& z_{1}=\xi_{1} \\
& z_{2}=\xi_{1} e^{1+\xi_{2} / \lambda} \\
& z_{3}=\frac{\xi_{2}}{c}+\frac{\xi_{2}^{2}}{c \lambda}+\frac{b}{c} e^{-\left(1+\xi_{2} / \lambda\right)}-d \xi_{2}^{2 / 3}-\frac{\xi_{3}}{c \lambda \xi_{1}} .
\end{aligned}
$$

It is easy to verify that $\Theta$ is a diffeomorphism and that both $\Theta$ and $\Theta^{-1}$ are uniformly Lipschitz in $D$, hence the GULDO hypothesis is verified.

Regards to the design of the gain $K$, Theorem 5 in [10] requires that $K$ is chosen in order to assign eigenvalues with negative real part to $A_{b}-K C_{b}$, where $\left(A_{b}, C_{b}\right)$ are a Brunowski pair. Let $\mathscr{L}$ be the set of eigenvalues of $A_{b}-K C_{b}$ and $\ell=\max \Re(\mathscr{L}))<0$. Parameter $\alpha$ can take value in $(0,-\ell)$ and it corresponds to the rate of exponential convergence to 0 of the observation error. For each choice of $\alpha$ there is a corresponding delay bound $\bar{\theta}$ and a larger value for $\alpha$ implies a smaller value for $\bar{\theta}$. The relationship between $\alpha$ and $\bar{\theta}$ depends on $K$ and on the Lipschitz constants of the system. Since $\bar{\theta}$ must be larger than the interval between any two consecutive measurements, this results is a constraint on $\alpha$, and the optimal choice of $K$ is the one that yields the largest $\alpha$ for a given $\bar{\theta}$. Notice that if $\bar{\theta}$ is too large no positive value of $\alpha$ can satisfy the constraint, in other words the sampling interval is too large for the observer to converge to the state of the system. Since the Lipschitz constants are difficult to determine, and the theoretical bounds are in any case only sufficient conditions, the choice of $\alpha$ and $K$ can be performed through off-line simulations for each value of $\bar{\theta}$ by using the model parameters. Numerical simulations have shown that a sampling interval $\bar{\theta} \in[0,3]$ days allows an appropriate choice of $K$ that guarantees good performance of the observer in less than 2 days, that is, only 1-2 measurements are sufficient. This is compatible with the experimental practice under consideration [27].

\section{Impulsive control synthesis}

The computation of the optimal control requires the Carleman discretization of the impulsive Hahnfeldt et al. model in each time interval $\left[\tau_{k}, \tau_{k+1}\right)$. The discretization is provided in the form (5)-(7) and is practically achieved by truncating the Taylor series in (7), thus obtaining a polynomial approximation for $F_{k}\left(x_{k}\right)$ (see [6], [7] for the details about the general case). In Table I the analytic expression of the first 4 coefficients of the Taylor series are reported.

The blocks $A_{j}^{i}(x)$ for $i=1$ defined in (9) assume the form

$$
\begin{gathered}
A_{0}^{1}(x)=f(x)=\left[\begin{array}{l}
f_{1}(x) \\
f_{2}(x) \\
f_{3}(x)
\end{array}\right]=\left[\begin{array}{c}
-\lambda x_{1} \ln \left(\frac{x_{1}}{x_{2}}\right) \\
b x_{1}-d x_{1}^{2 / 3} x_{2}-c x_{2} x_{3} \\
-\eta x_{3}
\end{array}\right] \\
A_{1}^{1}(x)=\left[\begin{array}{ccc}
-\lambda\left(1+\ln \left(\frac{x_{1}}{x_{2}}\right)\right) & \frac{\lambda x_{1}}{x_{2}} & 0 \\
b-\frac{2 d x_{2}}{3 x_{1}^{(1 / 3)}} & -c x_{3}-d x_{1}^{(2 / 3)} & -c x_{2} \\
0 & 0 & -\eta
\end{array}\right]
\end{gathered}
$$

TABLE I

COEFFICIENTS $P_{j}^{1}(x)$ OF THE TAYLOR SERIES FOR $j \leq 3$

\begin{tabular}{|c||c|}
\hline$j$ & $P_{j}^{1}(x)$ \\
\hline \hline 0 & $A_{0}^{1}=f(x)$ \\
\hline 1 & $A_{1}^{1} A_{0}^{1}=J(x) f(x)$ \\
\hline 2 & $\left(A_{1}^{1}\right)^{2} A_{0}^{1}+2 A_{2}^{1}\left(A_{0}^{1}\right)^{[2]}$ \\
\hline 3 & $\left(A_{1}^{1}\right)^{3} A_{0}^{1}+\left(2 A_{1}^{1} A_{2}^{1}+3 A_{2}^{1} A_{1}^{2}\right)\left(A_{0}^{1}\right)^{[2]}+6 A_{3}^{1}\left(A_{0}^{1}\right)^{[3]}$ \\
\hline \hline
\end{tabular}

$$
A_{2}^{1}(x)=\left[\begin{array}{ccccccccc}
\frac{-\lambda}{x_{1}} & \frac{\lambda}{x_{2}} & 0 & \frac{\lambda}{x_{2}} & \frac{-\lambda x_{1}}{x_{2}^{2}} & 0 & 0 & 0 & 0 \\
\frac{2 d x_{2}}{3 x_{1}^{(4 / 3)}} & \frac{-2 d}{3 x_{1}^{(1 / 3)}} & 0 & \frac{-2 d}{3 x_{1}^{(1 / 3)}} & 0 & -c & 0 & -c & 0 \\
0 & 0 & 0 & 0 & 0 & 0 & 0 & 0 & 0
\end{array}\right],
$$

whereas (10) is used for $i>1$, for example

$$
A_{0}^{2}=A_{0}^{1} \otimes I_{3}+I_{3} \otimes A_{0}^{1}
$$

and its explicit form is

$$
A_{0}^{2}=\left[\begin{array}{ccc}
2 f_{1}(x) & 0 & 0 \\
f_{2}(x) & f_{1}(x) & 0 \\
f_{3}(x) & 0 & f_{1}(x) \\
f_{2}(x) & f_{1}(x) & 0 \\
0 & 2 f_{2}(x) & 0 \\
0 & f_{3}(x) & f_{2}(x) \\
f_{3}(x) & 0 & f_{1}(x) \\
0 & f_{3}(x) & f_{2}(x) \\
0 & 0 & 2 f_{3}(x)
\end{array}\right]
$$

It can be noticed that the recursive nature of (10) makes extremely easy the automatic computation of higher order terms.

Once the Carleman discretization allows to restate the optimal control problem in a pure discrete-time framework, equations (11) have to be solved. To this end it is necessary to compute the derivatives of $\Psi_{k}$ with respect to $v_{k+1}$ and $x_{k}$. The former is immediately obtained as $\nabla_{v_{k+1}} \otimes \Psi_{k}=B$. The latter can be computed as

$$
\nabla_{x_{k}} \otimes \Psi_{k}=I_{n}+\sum_{j=0}^{\infty}\left(\nabla_{x_{k}} \otimes P_{j}^{1}\left(x_{k}\right)\right) \frac{\theta_{k}^{j+1}}{(j+1) !} .
$$

Notice that, due to the particular structure of the impulsive system (1), $\nabla_{v_{k+1}} \otimes \Psi_{k}$ does not depend on $x_{k}$ (it is constant, actually) and $\nabla_{x_{k}} \otimes \Psi_{k}$ does not depend on $v_{k+1}$.

It is useful for the applications to have $\nabla_{x_{k}} \otimes \Psi_{k}$ in (33) expressed as a recursive function of the blocks $A_{j}^{1}$. To this end, the following Lemmas are required (see [7] for the proofs and more details).

Lemma 1: For any $j \geq 0$ and $i>0$ it is:

$$
\begin{aligned}
\nabla_{x} \otimes A_{j}^{i}= & (j+1)\left(A_{j+1}^{1} \otimes I_{n}^{[i-1]}\right) \\
& +\left[I_{n} \otimes \mathcal{A}_{1}^{i-1, j}, \ldots I_{n} \otimes \mathcal{A}_{n}^{i-1, j}\right]
\end{aligned}
$$

with

$$
\mathcal{A}_{l}^{i, j}=\nabla_{x_{l}} \otimes A_{j}^{i}=\frac{\partial A_{j}^{i}}{\partial x_{l}}
$$

and $x_{l}$ is the $l$-th component of vector $x$. In case of $i=1$ :

$$
\nabla_{x} \otimes A_{j}^{1}=(j+1) A_{j+1}^{1}
$$


Lemma 2: For $j \geq 0 \nabla_{x} \otimes P_{j}^{1}$ admits the expression

$$
\begin{aligned}
& \nabla_{x} \otimes P_{0}^{1}=A_{1}^{1} \\
& \nabla_{x} \otimes P_{j}^{1}=\sum_{l=1}^{j}(l+1) A_{l+1}^{1}\left(I_{n} \otimes P_{j-1}^{l}\right)+A_{l}^{1}\left(\nabla_{x} \otimes P_{j-1}^{l}\right)
\end{aligned}
$$

and, for $i>1, \nabla_{x} \otimes P_{j}^{i}$ is given by

$\nabla_{x} \otimes P_{j}^{i}=\sum_{l=0}^{j-i+1}\left[\left(\nabla \otimes A_{l}^{i}\right)\left(I_{n} \otimes P_{j-1}^{l+i-1}\right)+A_{l}^{i}\left(\nabla_{x} \otimes P_{j-1}^{l+i-1}\right)\right]$

Since $\nabla_{x} \otimes P_{0}^{1}=\nabla_{x} \otimes f=A_{1}^{1}$ is the Jacobian, this analysis shows that each term $\nabla_{x} \otimes P_{j}^{1}$ in (33) is computable from the matrices $A_{j}^{1}$, e.g. the ones reported in (28)-(30).

The design of the optimal control is completed by specifying the matrices $Q_{N}$ and $R_{k}$ in the optimization index (4). The choice of the weight matrices is done according to the following aims:

- bring the tumor volume within a specified range of the final reference level $\bar{x}$ at the end of the prescribed timehorizon;

- keep the average daily amount of the administered drug below a given bound;

- minimize the total amount of the administered drug in the prescribed time-horizon.

\section{PERFormance ANALYSis}

In order to obtain an effective benchmark for the proposed impulsive drug administration therapy we build up a population of 500 virtual subjects. Each subject (mice, actually) is associated to a set of the Hahnfeldt et al. model parameters. These model parameters are sampled from a multivariate Gaussian distribution, whose average value and covariance matrix are identified from experimental data taken from the available literature, eventually providing a population of rather heterogenous individuals. The control law and observer parameters are fixed once and for all the virtual mice: they are set by simulations on the ground of the average virtual mouse and do not vary when applied to the population individuals, thus allowing to test a posteriori the robustness of the algorithm.

\section{A. Building of virtual mice population}

The starting point to build up an in silico validation of the proposed anti-angiogenic tumor control law is to properly define a population of virtual subjects onto apply the drug administration therapy. To this end we exploited experimental data taken from [27] where different types of human cancer cells were implanted in the s.c. dorsa of mice. More specifically, we obtained data from Figures 1.a, 2.a and 2.d of [27], where tumor volume evolutions (BxPC-3 tumor) are reported over a time horizon spanning from 25 to 35 days (with an inter-sampling period spanning from 2 to 5 days), with or without a prescribed therapy. In summary we got streams of data for two sets of 14 mice each, one set referring to the control group (i.e. without any therapy), the other referring to the therapy group (i.e. under endostatin administration).
The Hahnfeldt et al. model parameters have been identified according to the following double-step procedure. First, the control group is exploited, allowing to estimate the triple $(\lambda$, $b, d)$ for each mouse; average value and standard deviation over the 14 mice control group are reported in Table II. Then, the other pair of parameters related to the drug administration (i.e. $c$ and $\eta$ ) are estimated from the therapy group, with the Gompertz growth parameters $(\lambda, b, d)$ provided to the model by the aforementioned average values. In this case we found a negligible variability within the mice population, thus we did not report any variance in Table II. It worths noticing that the whole set of parameters could not be identified in a unique step since the therapy group does not allow to identify the Gompertz growth parameters, due to identification problems.

TABLE II

POPULATION OF VIRTUAL MICE: HAHNFELDT et al. MODEL PARAMETERS

\begin{tabular}{|c|c|c|c|}
\hline Parameter & Unit & Mean & Std \\
\hline$\lambda$ & {$\left[\right.$ day $\left.^{-1}\right]$} & 0.0735 & 0.0144 \\
\hline$b$ & {$\left[\right.$ day $\left.^{-1}\right]$} & 4.0501 & 2.4906 \\
\hline$d$ & {$\left[\right.$ day $\left.^{-1} \mathrm{~mm}^{-2}\right]$} & 0.0097 & 0.0041 \\
\hline$c$ & {$\left[\right.$ day $\left.^{-1}(\mathrm{mg} / \mathrm{kg})^{-1}\right]$} & 0.0955 & 0.0 \\
\hline$\eta$ & {$\left[\right.$ day $\left.^{-1}\right]$} & 0.6240 & 0.0 \\
\hline
\end{tabular}

The mice population exploited to validate the control law is generated by randomly sampling triples of Gompertz growth parameters $(\lambda, b, d)$ from a multivariate Gaussian distribution with average values (reported in Table II) and covariance matrix provided by the identification procedure (we report only standard deviations in Table II). Parameters $(c, \eta)$ are, instead, fixed for all virtual mice. Initial conditions for $z_{1}$ and $z_{2}$ are sampled from independent Gaussian random variables with average values 100 and 200, respectively, and a Coefficient of Variation (CV) of $20 \%$ for both. the initial condition for $z_{3}$ is fixed equal to 0 .

\section{B. Control law implementation}

Motivated by the fact that often measurements are more difficult, time-consuming and expensive to obtain than control actuation, simulations reported comply with the Control Algorithm A2 reported in Section II-C, with less measurements than control impulses.

According to the clinical literature [25], [27], the desired final tumor size is set equal to $50 \mathrm{~mm}^{3}$, i.e. half of the initial size. The desired final value for the carrying capacity has been set to $1 \mathrm{~mm}^{3}$, which is a reasonable approximation of $\epsilon_{z}$.

We do not specify any desired final value for the level of the administered angiogenic inhibitor (i.e. $z_{3}$ ).

Regards to the observer parameters, the gain $K$ is designed in order to place the eigenvalues of $A_{b}-K C_{b}$ at $\{-0.1,-0.2,-0.3\}$. This choice is fixed for all simulations on the virtual mice population. Measurements are available once a day, thus we assume to have a fixed inter-sampling period $s_{m+1}-s_{m}=1$ day, $\forall m=1, \ldots, N^{\prime}$.

Regards to the Carleman discretization step, we made simulations with different truncations of the Taylor series (up to including 6th order terms): simulations showed that after 
the 4th order term, to include further terms in the summation does not provide meaningful improvements.

Finally the weight matrices $Q_{N}$ and $R_{k}$ of the cost function (4), are set equal to $\operatorname{diag}\{450,225,0\}$ and 50, respectively. Notice that the last element of $Q_{N}$ is set equal to zero because the cost for $z_{3}$ is already included in the cost of the input. The remaining parameters of $Q_{N}$ and $R_{k}$ are chosen to balance the final tumor size and the amount of drug infusion with a heuristic approach on simulations on the average mouse. The impulsive control action happens 4 times per day on a timehorizon of 13 days. The discrete time unit is therefore 0.25 days, that is, $\tau_{k+1}-\tau_{k}=0.25$ for all $k$, and the time horizon is $N=52$ with a control impulse per time unit. The length of the receding horizon is $M=4$ time units that corresponds to 1 day.

\section{Simulation results and discussion}

Besides the primal regulatory issue concerning tumor volume reduction, further secondary issues have to be properly accounted for when evaluating the control law performances. For instance we need to supervise the daily maximum dose administered during the treatment. Therefore, we evaluated the goodness of the proposed control law in terms of the following items:

- how close to the reference level is the final tumor volume (i.e. $z_{1}(t=13$ days));

- daily upper bound of the administered drug.

We actually tighten this second issue by monitoring each single drug administration to be possibly lower than $25 \mathrm{mg} / \mathrm{kg}$ (that ensures a daily drug administration lower than 100 $\mathrm{mg} / \mathrm{kg} /$ day, since we have 4 impulses per day).

We assume to know the initial estimates for $z_{1}$ and $z_{3}$. Indeed $z_{1}(0)$, the initial tumor mass, can be measured before the drug administration therapy starts, while $z_{3}(0)$, the initial level of administered drug, is known to be at zero in absence of a therapy. Instead, we assign the initial estimate of the carrying capacity as a Gaussian random variable with average value $150 \mathrm{~mm}^{3}$ (25\% less than the real value) and a CV $=20 \%$.

Figure 1 reports the time evolution for the three state variables, endowed with their estimates, for the average virtual mouse. It can be appreciated how good the actual state is estimated by the observer (the carrying capacity is clamped before the first day of therapy). Moreover, the final value of the tumor size reaches the required target values at the end of the treatment.

Figure 2 show that actual control impulses for a sample of 5 virtual mice randomly extracted from the population. It can be appreciated that the actual input is not uniform and it varies across subjects as a consequence of the unknown parameters used to generate each mouse. The amount of daily drug administration is never above the threshold of $100 \mathrm{mg} / \mathrm{kg} / \mathrm{day}$, whereas the first dose is occasionally larger than $25 \mathrm{mg} / \mathrm{kg}$, whci is a reasonable behavior.

Numerical simulations show that $100 \%$ of the population reduces the tumor size with respect to the initial condition. Table III describes how close is the final tumor mass to the desired one. For instance, it is apparent that in the $80 \%$ of cases the final tumor size is smaller than $60 \mathrm{~mm}^{3}, 20 \%$ greater than the desired value.

TABLE III

CONTROL LAW PERFORMANCE

\begin{tabular}{|c|c|c|c|c|c|}
\hline Final tumor value $\left[\mathrm{mm}^{3}\right]$ & $<50$ & $<55$ & $<60$ & $<65$ & $<70$ \\
\hline Percentage & $35 \%$ & $65 \%$ & $80 \%$ & $91 \%$ & $98 \%$ \\
\hline
\end{tabular}

Figure 3 collects data from the closed-loop simulations on the population of virtual mice according to a grid that aims at resembling the Control Variability Grid Analysis recently exploited in the different medical framework of the artificial pancreas (see, e.g. [39]). The $x$-axis refers to the final tumor value and the $y$-axis reports the maximum among each single drug administration. We may divide the grid into four zones. The light green one refers to virtual subjects of the population that are very good controlled (their final tumor mass is within $10 \%$ of the desired target value) according to an impulse drug delivery that never exceeds the chosen upper bound of $25 \mathrm{mg} / \mathrm{kg}$ per single impulse $(63 \%)$. It can be appreciated it is the majority of the 500 individuals. The dark green refers to virtual mice that are satisfactory controlled (their final tumor mass is within $40 \%$ of the desired target value) according to an impulse drug delivery that never exceeds the chosen upper bound of $25 \mathrm{mg} / \mathrm{kg}$ per single impulse. Notice that the light and dark green zones include the great majority of the population $(95 \%)$. The orange zone refers to individuals that are satisfactory controlled, though according to a single dose that exceeds (at least one time) the bound of $25 \mathrm{mg} / \mathrm{kg}$. The rest of the grid (red zones) refer to individuals whose final tumor mass is more than $40 \%$ greater than the desired one, or that allow a maximum control impulse that exceeds $50 \mathrm{mg} / \mathrm{kg}$. They constitute a very small percentage (3.4\%).

\section{CONCLUSIONS}

One of the key problems in medical oncology is the fact that tumor size measurements and other parameters are achieved on a very irregular basis. In addition the treatment delivery usually happens periodically in the form of control impulses. This situation appears to be quite common in the clinical practice and, consequently, the traditional approaches to the outputfeedback control problem need to be substantially modified in order to be of help in this area. In this work we have presented an output feedback control framework based on sparse measurements and controls that appears promising for developing model-based protocols. We have validated our approach on a model-based feedback antiangiogenic tumor therapy, where a separation results allows to separately estimate the unknown parameters of the model from the sparse measurements and to design an advanced discretization-based optimal impulsive control strategy. Our results show that for the chosen model the approach can efficiently cope with un-modeled parameter uncertainties under reasonable constraints on the frequency of measurements and controls. From a quantitative perspective the results seem consistent with recently published results about the optimal control of antiangiogenic therapies under the simplified assumption that the system state and the model 


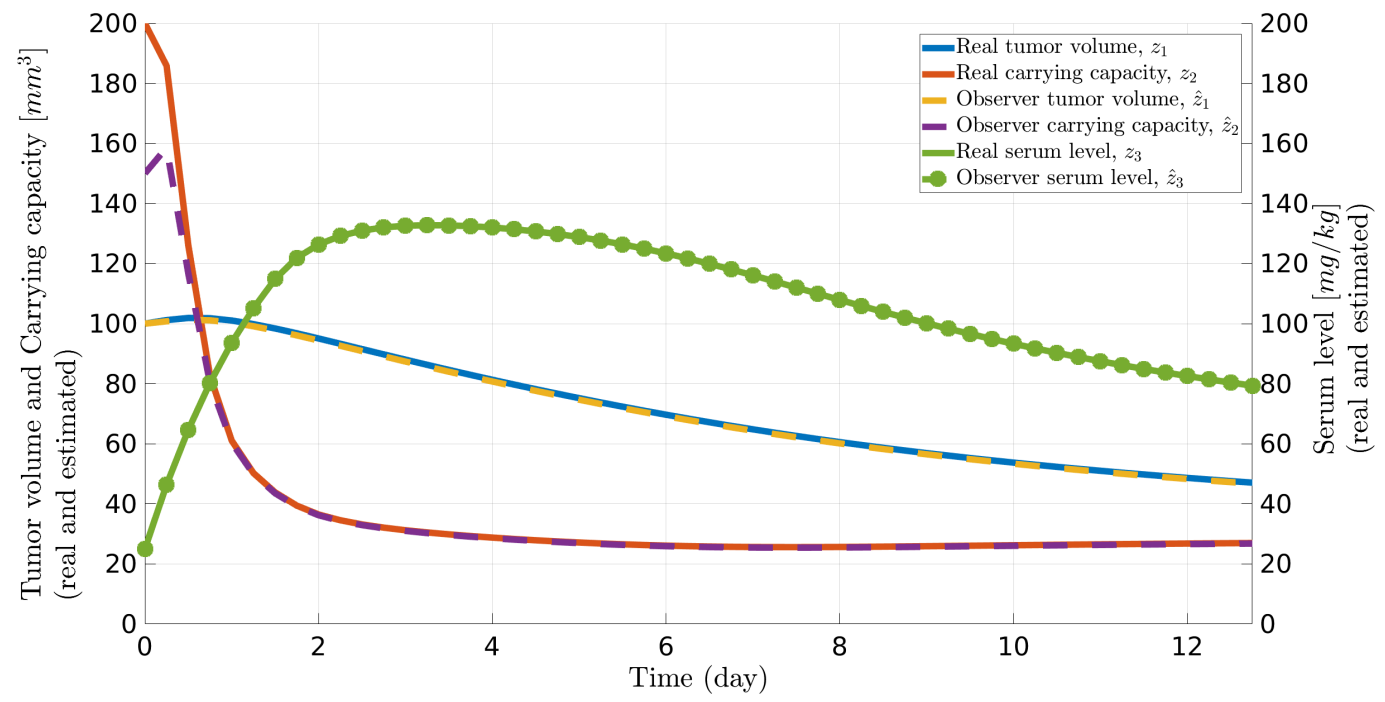

Fig. 1. Time evolution for the state variables of the closed-loop system for the average virtual mouse. The $y$-axis reports $z_{1}$ and $z_{2}$ in [mm $\left.{ }^{3}\right]$ as well as $z_{3}$ in $[\mathrm{mg} / \mathrm{kg}]$.

parameters are known [17]. Our work can be further extended by deriving optimal control laws for different choices of the cost function and for more sophisticated constraints on the therapy that may arise in the clinical practice. Another interesting point that deserves further investigation is the application of the proposed framework to the optimal time scheduling of therapies [20].

\section{ACKNOWLEDGMENT}

The authors wish to thank the anonymous reviewers for their valuable suggestions that greatly improved the quality of the paper. The authors also thank Prof. Alfredo Germani for his contribution to the development of the methodological framework of this work.

\section{REFERENCES}

[1] A. Abdollahi, K.E. Lipson, A. Sckell, H. Zieher, F. Klenke, D. Poerschke, A. Roth, X. Han, M. Krix, M. Bischof, et al. Combined therapy with direct and indirect angiogenesis inhibition results in enhanced antiangiogenic and antitumor effects. Cancer research, 63(24):88908898, 2003.

[2] Z. Agur, L. Cojocaru, G. Mazor, and Y.L. Anderson, R.M.and Danon. Pulse mass measles vaccination across age cohorts. Proceedings of the National Academy of Sciences, 90(24):11698-11702, 1993.

[3] N. Bellomo and L. Preziosi. Modelling and mathematical problems related to tumor evolution and its interaction with the immune system. Mathematical and Computer Modelling, 32(3-4):413-452, 2000.

[4] T. Boehm, J. Folkman, T. Browder, and M.S. O'Reilly. Antiangiogenic therapy of experimental cancer does not induce acquired drug resistance. Nature, 390(6658):404, 1997.

[5] T. Browder, C.E. Butterfield, B.M. Kräling, B. Shi, B. Marshall, M.S O'Reilly, and J. Folkman. Antiangiogenic scheduling of chemotherapy improves efficacy against experimental drug-resistant cancer. Cancer research, 60(7):1878-1886, 2000.

[6] F. Cacace, V. Cusimano, and A. Germani. An efficient approach to the design of observers for continuous-time systems with discrete-time measurements. In IEEE 50th Conference on Decision and Control and European Control Conference (CDC-ECC), pages 7549-7554. IEEE, 2011.
[7] F. Cacace, V. Cusimano, A. Germani, and P. Palumbo. Carleman discretization of impulsive systems: application to the optimal control problem of anti-angiogenic tumor therapies. In IEEE 55th Conference on Decision and Control (CDC), pages 1042-1047, 2016.

[8] F. Cacace, V. Cusimano, A. Germani, P. Palumbo, and P. Papa. Closedloop control of tumor growth by means of anti-angiogenic administration. to appear on Mathematical Biosciences and Engineering, 15(4), 2018.

[9] F. Cacace, A. Germani, and C. Manes. An exponential observer with delay-dependent gain for a class of nonlinear systems with time-varying measurement delay. In IEEE 51st Conference on Decision and Control $(C D C)$, pages 2364-2369. IEEE, 2012.

[10] F. Cacace, A. Germani, and C. Manes. A chain observer for nonlinear systems with multiple time-varying measurement delays. SIAM Journal on Control and Optimization, 52(3):1862-1885, 2014.

[11] A. d'Onofrio. Stability properties of pulse vaccination strategy in seir epidemic model. Mathematical biosciences, 179(1):57-72, 2002.

[12] A. d'Onofrio and A. Gandolfi. Tumour eradication by antiangiogenic therapy: analysis and extensions of the model by hahnfeldt et al.(1999). Mathematical biosciences, 191(2):159-184, 2004.

[13] A. d'Onofrio and A. Gandolfi. A family of models of angiogenesis and anti-angiogenesis anti-cancer therapy. Mathematical medicine and biology: a journal of the IMA, 26(1):63-95, 2008.

[14] A. d'Onofrio and A. Gandolfi. Chemotherapy of vascularised tumours: role of vessel density and the effect of vascular "pruning". Journal of theoretical biology, 264(2):253-265, 2010.

[15] A. d'Onofrio and A. Gandolfi. Resistance to antitumor chemotherapy due to bounded-noise-induced transitions. Physical Review E, 82(6):061901, 2010

[16] A. d'Onofrio, U. Ledzewicz, H. Maurer, and H. Schättler. On optimal delivery of combination therapy for tumors. Mathematical biosciences, 222(1):13-26, 2009.

[17] D.A. Drexler, J. Sápi, and L. Kovács. Optimal discrete time control of antiangiogenic tumor therapy. In Proceedings of the 20th IFAC World Congress, pages 14046-14051, 2017.

[18] D.A. Drexler, J. Sápi, and L. Kovács. Positive nonlinear control of tumor growth using angiogenic inhibition. In Proceedings of the 20th IFAC World Congress, pages 15633-15638, 2017.

[19] J.P. Eder Jr, J.G. Supko, J.W. Clark, T.A. Puchalski, R. GarciaCarbonero, D.P. Ryan, L.N. Shulman, J. Proper, M. Kirvan, B. Rattner, et al. Phase I clinical trial of recombinant human endostatin administered as a short intravenous infusion repeated daily. Journal of Clinical Oncology, 20(18):3772-3784, 2002.

[20] A. Ergun, K. Camphausen, and L.M. Wein. Optimal scheduling of radiotherapy and angiogenic inhibitors. Bulletin of mathematical biology, 65(3):407-424, 2003. 

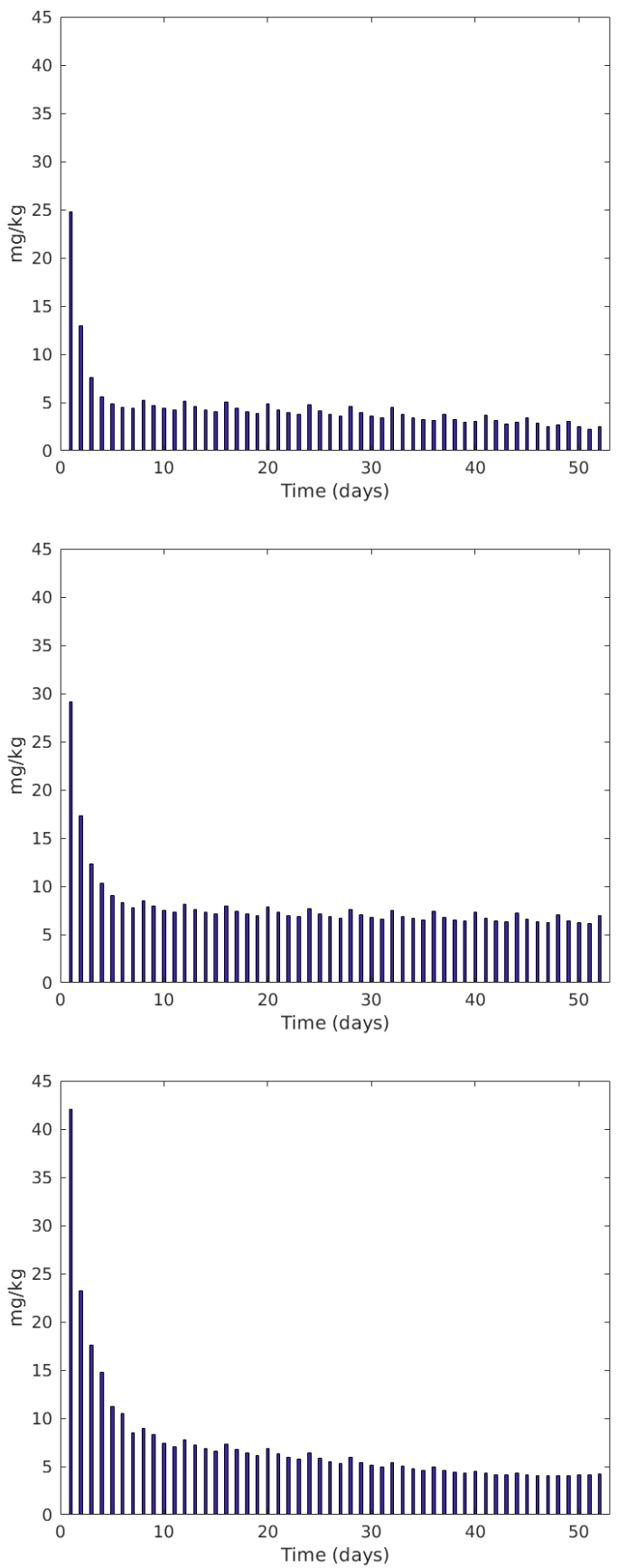

Fig. 2. Control impulses for a random sample of 5 virtual mice.

[21] J. Folkman. Tumor angiogenesis: therapeutic implications. New England journal of medicine, 285(21):1182-1186, 1971.

[22] J. Folkman. Angiogenesis inhibitors: a new class of drugs. Cancer biology \& therapy, 2(sup1):126-132, 2003.

[23] J. Folkman. Antiangiogenesis in cancer therapyendostatin and its mechanisms of action. Experimental cell research, 312(5):594-607, 2006.

[24] S.L. Fraga and F.L. Pereira. Hamilton-Jacobi-Bellman equation and feedback synthesis for impulsive control. IEEE Transactions on Automatic Control, 57(1):244-249, 2012.

[25] P. Hahnfeldt, D. Panigrahy, J. Folkman, and L. Hlatky. Tumor development under angiogenic signaling. Cancer research, 59(19):4770-4775, 1999.

[26] R.S. Kerbel. Improving conventional or low dose metronomic chemotherapy with targeted antiangiogenic drugs. Cancer research and
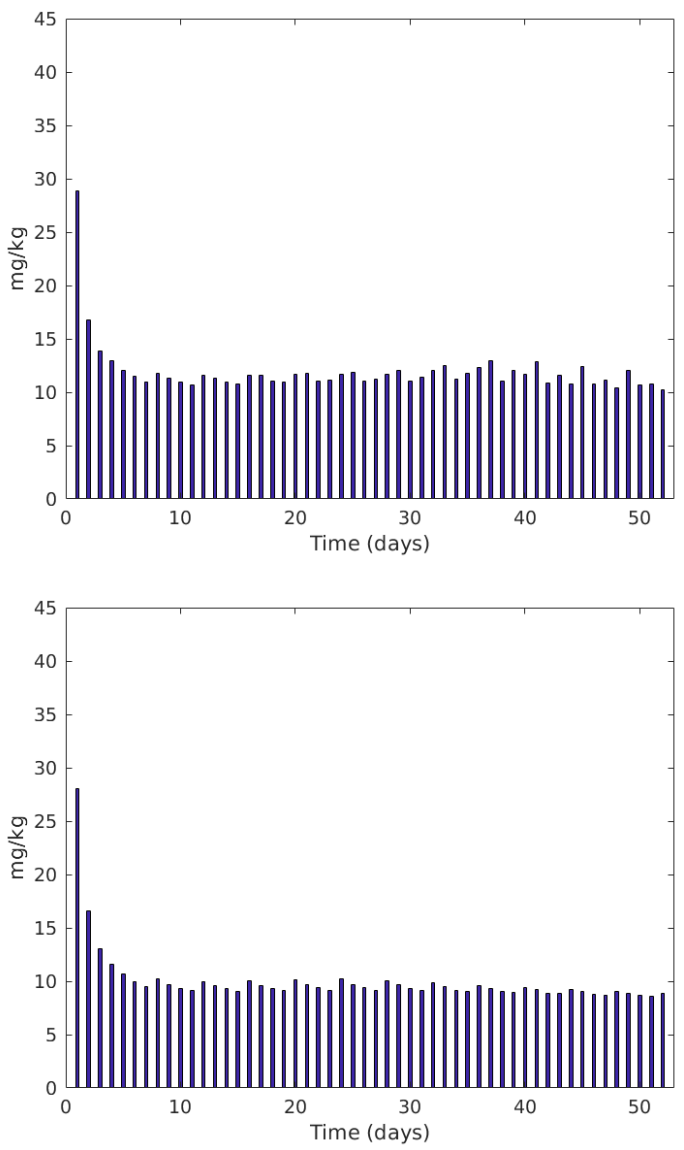

treatment: official journal of Korean Cancer Association, 39(4):150, 2007.

[27] O. Kisker, C.M. Becker, D. Prox, M. Fannon, R. DAmato, E. Flynn, W.E. Fogler, B.K.L. Sim, E.N. Allred, S.R. Pirie-Shepherd, et al. Continuous administration of endostatin by intraperitoneally implanted osmotic pump improves the efficacy and potency of therapy in a mouse xenograft tumor model. Cancer Research, 61(20):7669-7674, 2001.

[28] J. Klamka and A Świerniak. Controllability of a model of combined anticancer therapy. Control and Cybernetics, 42(1):123-138, 2013.

[29] K. Kowalski and W.-H. Steeb. Nonlinear dynamical systems and Carleman linearization. World Scientific, 1991.

[30] M.H. Kulke, E.K. Bergsland, D.P. Ryan, P.C. Enzinger, T.J. Lynch, A.X. Zhu, J.A. Meyerhardt, J.V. Heymach, W.E. Fogler, C. Sidor, et al. Phase II study of recombinant human endostatin in patients with advanced neuroendocrine tumors. Journal of Clinical Oncology, 24(22):3555- 


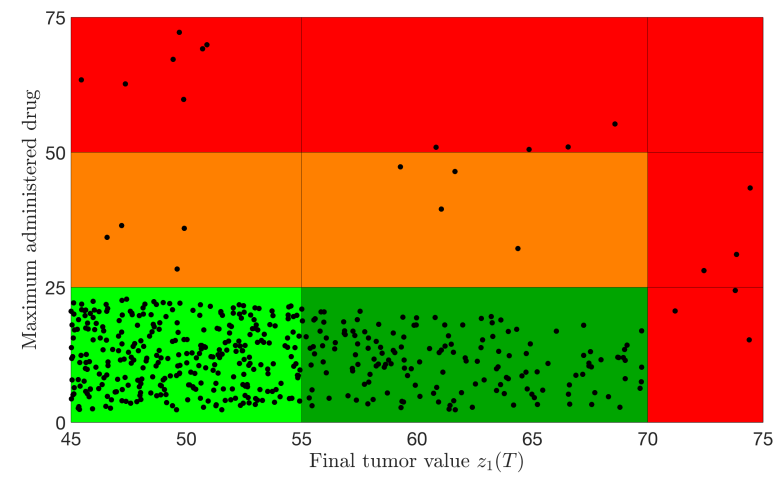

Fig. 3. Grid analysis for the population.

$3561,2006$.

[31] V. Lakshmikantham, D.D. Bainov, and P.S. Simeonov. Theory of impulsive differential equations, volume 6. World scientific, 1989.

[32] U. Ledzewicz, H. Maurer, and H. Schaettler. Optimal and suboptimal protocols for a mathematical model for tumor anti-angiogenesis in combination with chemotherapy. Mathematical Biosciences and Engineering, 8(2):307-323, 2011.

[33] U. Ledzewicz, H. Maurer, and H. Schättler. Bang-bang and singular controls in a mathematical model for combined anti-angiogenic and chemotherapy treatments. In Proceedings of the 48th IEEE Conference on Decision and Control, held jointly with the 28th Chinese Control Conference. CDC/CCC, pages 2280-2285. IEEE, 2009.

[34] U. Ledzewicz and H. Schättler. Antiangiogenic therapy in cancer treatment as an optimal control problem. SIAM Journal on Control and Optimization, 46(3):1052-1079, 2007.

[35] U. Ledzewicz and H. Schättler. Analysis of optimal controls for a mathematical model of tumour anti-angiogenesis. Optimal Control Applications and Methods, 29(1):41-57, 2008.

[36] U. Ledzewicz and H. Schättler. Optimal and suboptimal protocols for a class of mathematical models of tumor anti-angiogenesis. Journal of Theoretical Biology, 252(2):295-312, 2008.

[37] Z.G. Li, C.Y. Wen, and Y.C. Soh. Analysis and design of impulsive control systems. IEEE Transactions on Automatic Control, 46(6):894897, 2001.

[38] G. Limaverde-Sousa, C. Sternberg, and C. G. Ferreira. Antiangiogenesis beyond vegf inhibition: a journey from antiangiogenic single-target to broad-spectrum agents. Cancer treatment reviews, 40(4):548-557, 2014.

[39] L. Magni, D.M. Raimondo, C. Dalla Man, M. Breton, S. Patek, G. De Nicolao, C. Cobelli, and B. Kovatchev. Evaluating the efficacy of the closed-loop glucose regulation via control-variability grid analysis. J Diabetes Sci Technol., 2:630-635, 2008.

[40] R. Missiaen, F.M. Rodriguez, G. Eelen, and P. Carmeliet. Targeting endothelial metabolism for anti-angiogenesis therapy: a pharmacological perspective. Vascular pharmacology, 2017.

[41] M. Motta and F. Rampazzo. Dynamic programming for nonlinear systems driven by ordinary and impulsive controls. SIAM Journal on Control and Optimization, 34(1):199-225, 1996.

[42] M.S. O'Reilly, T. Boehm, Y. Shing, N. Fukai, G. Vasios, W.S. Lane, E. Flynn, J.R. Birkhead, B. R Olsen, and J. Folkman. Endostatin: an endogenous inhibitor of angiogenesis and tumor growth. Cell, 88(2):277-285, 1997

[43] M.S. O'Reilly, L. Holmgren, Y. Shing, C. Chen, R.A. Rosenthal, M. Moses, W.S. Lane, Y. Cao, E.H. Sage, and J. Folkman. Angiostatin: a novel angiogenesis inhibitor that mediates the suppression of metastases by a lewis lung carcinoma. Cell, 79(2):315-328, 1994.

[44] F.L. Pereira and G.N. Silva. Necessary conditions of optimality for vector-valued impulsive control problems. Systems \& Control Letters, 40(3):205-215, 2000

[45] J. Sápi, D.A. Drexler, I. Harmati, Z. Sápi, and L. Kovács. Qualitative analysis of tumor growth model under antiangiogenic therapy-choosing the effective operating point and design parameters for controller design. Optimal Control Applications and Methods, 37(5):848-866, 2016.

[46] J. Sápi, L. Kovács, D.A. Drexler, P. Kocsis, D. Gajári, and Z. Sápi. Tumor volume estimation and quasi-continuous administration for most effective bevacizumab therapy. PloS one, 10(11):e0142190, 2015.
[47] H. Schättler and U. Ledzewicz. Optimal control for mathematical models of cancer therapies - An application of geometric methods. Springer, 2015.

[48] B. Shulgin, . Stone, and Z. Agur. Pulse vaccination strategy in the sir epidemic model. Bulletin of mathematical biology, 60(6):1123-1148, 1998.

[49] G.N. Silva and R.B. Vinter. Necessary conditions for optimal impulsive control problems. SIAM Journal on Control and Optimization, 35(6):1829-1846, 1997

[50] I. Stamova and G.T. Stamov. Applied impulsive mathematical models. Springer, 2016

[51] J. Sun, Y. Zhang, and O. Wu. Less conservative conditions for asymptotic stability of impulsive control systems. IEEE Transactions on Automatic Control, 48(5):829-831, 2003.

[52] A. Świerniak and J. Klamka. Comparison of controllability conditions for models of antiangiogenic and combined anticancer therapy. IFAC Proceedings Volumes, 47(3):11530-11535, 2014.

[53] P.R. Wolenski and S. Žabić. A sampling method and approximation results for impulsive systems. SIAM Journal on Control and Optimization, 46(3):983-998, 2007.

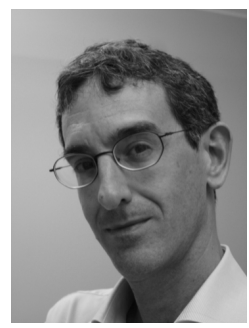

Filippo Cacace graduated in electronic engineering at Politecnico di Milano in 1988, where he received the $\mathrm{PhD}$ degree in computer science in 1992. Since 2003 he has been working with Centro Integrato di Ricerca at University Campus Biomedico of Rome, where he is currently an associate professor. His current research interests include nonlinear systems and observers, stochastic and delay systems, system identification, and applications to systems biology.

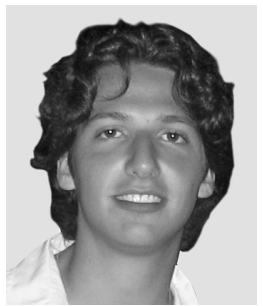

Valerio Cusimano received the Laurea degree in 2008 and the Ph.D. degree in 2012, both in biomedical engineering from the University Campus BioMedico of Rome. In 2012 he joined the Istituto di Analisi dei Sistemi e Informatica "A. Ruberti" of the Italian National Research Council, Rome, where he is currently a researcher. The areas of interest are nonlinear filtering and control systems, mathematical modeling and control of tumor growth system, and systems biology.

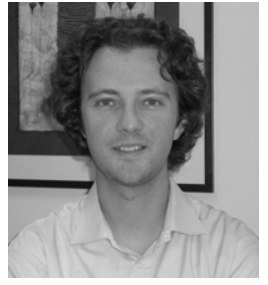

Pasquale Palumbo received the Laurea degree cum laude in 1995 and the Ph.D. degree in 2000, both in electrical engineering from the University of L'Aquila. In 2000 he joined the Istituto di Analisi dei Sistemi e Informatica "A. Ruberti" of the Italian National Research Council, Rome, where he is researcher since 2005. Since 2000 he has been a visiting professor at the University of L'Aquila, teaching courses on Probability, Systems and Control Theory and Systems Biology (this last since 2008). He is a member of the IEEE Control Systems Society and of the IEEE Technical Committee on Systems Biology and on Healthcare and Medical Systems. He is the author of about 100 peer-reviewed publications in the areas of nonlinear filtering and control systems, mathematical modeling and control of the glucose-insulin system, and systems biology. 